\title{
Assessing the Performance of a Medical Intensive Care Unit: A 5-year single-center Experience
}

\author{
Amir Anushiravani, Seyed Masoom Masoompour ${ }^{1}$ \\ Digestive Disease Research Center, Shariati Hospital, Tehran University of Medical Sciences, Tehran, ${ }^{1}$ Noncommunicable Diseases Research Center, \\ Shiraz University of Medical Sciences, Shiraz, Iran
}

\section{Abstract}

Patient care in the Intensive Care Unit (ICU) is complex and expensive, serving to provide optimal outcome as well as the adequate use of resources. Our objective was to determine variables associated with admission practices, processes of care, and clinical outcomes for critically ill patients. Admission records of a 10-bed ICU were gathered during a 5-year period. Variables such as average length of stay, bed turnover, bed occupancy rate, and turnover interval were evaluated. Of the 1719 patients evaluated, $54 \%$ were men. Mortality was highest between 10 pm and $2 \mathrm{am}$. There was no significant difference in ICU mortality during different days of the week. We showed that nonoffice hour admissions were not associated with poorer clinical outcomes, and significant differences in ICU mortality and ICU length of stay were not seen. Moreover, hospital mortality rates were not significantly higher for patients admitted to our ICU on weekends, at nights, or any day of the week.

Keywords: Intensive Care Unit, mortality, performance

\section{INTRODUCTION}

The Intensive Care Unit (ICU) is a place where physicians and nurses provide continuous monitoring and life support care. Despite improving patients' outcomes, there is still a relatively high mortality rate in ICU ward. Patient care in the ICU is complex and has high costs. The care of patients should ultimately serve as to provide optimal outcome as well as the adequate use of resources.

In addition, length of stay standardized for severity and type of illness and unplanned readmission rates are important outcomes; the classic outcome variable is mortality rate. The costs per surviving patient could also be an extremely important outcome variable.

The appropriate timelines of care and availability of complex tests and therapy are even more important in the first hours after admission to an ICU, when patients are more likely to be unstable and require extreme resuscitative measures. Although admission of patients who are unstable to ICUs occurs $24 \mathrm{~h}$ a day, not all ICUs maintain the same level of staffing during off-hours, that is, during nighttime, weekends, and holidays. A number of studies have demonstrated that

\begin{tabular}{|l|l|}
\hline \multicolumn{3}{|c|}{ Access this article online } \\
\hline Quick Response Code: & Website: \\
& www.ijccm.org \\
\cline { 2 - 2 } & \\
\hline
\end{tabular}

ICUs with mandatory intensivist consultation or closed ICU are associated with reduced mortality when compared with units with low-intensity physician staffing. ${ }^{[1]}$

Patients admitted in ICUs during off-hours may face worse outcomes. Several cohorts have shown an increased risk of death for patients admitted during off-hours, ${ }^{[2,3]}$ while others showed a surprising protective effect. ${ }^{[4,5]}$

Among various approaches, Pabon Lasso's (PL) model has proved itself to be one of the most useful models in assessing performance in patient health services. This model makes an overall assessment of hospital performance with the use of three indices; bed turnover (BTO), bed occupancy rate (BOR), and average length of stay (ALS). ${ }^{[6]}$

Our objective was to compare different variables concerning ICU performance and mortality to optimize ICU performance and patient care.

Address for correspondence: Dr. Seyed Masoom Masoompour, Shiraz University of Medical Sciences, P.0. Box 7193634154, Shiraz, Iran. E-mail:masoomm@sums.ac.ir

This is an open access article distributed under the terms of the Creative Commons Attribution-NonCommercial-ShareAlike 3.0 License, which allows others to remix, tweak, and build upon the work non-commercially, as long as the author is credited and the new creations are licensed under the identical terms.

For reprints contact: reprints@medknow.com

How to cite this article: Anushiravani A, Masoompour SM. Assessing the performance of a medical intensive care unit: A 5-year single-center experience. Indian J Crit Care Med 2017;21:163-6. 


\section{Case Report}

From March 2008 to March 2012, all admission records of 10-bed medical ICU of a teaching general hospital with 358 beds ${ }^{[7]}$ were retrieved from hospital information system (HIS). The outputs of HIS included the chart and card numbers, age, gender, date of admission and discharge, and outcome. To evaluate the ICU performance during this 5-year period, we calculated the ALS, BTO, BOR, and turnover interval (TI) through the following definitions and formula. ALS refers to the average number of days that a patient stays in a hospital. It is calculated by dividing the inpatient days to the number of admissions. BTO is a measure of productivity hospital beds and represents the number of patients treated per bed in a defined period of time (usually a year). We calculated this index by dividing total admissions into the number of beds. BOR indicates the percentage of beds occupied by patients in a defined period of time, usually a year. $\mathrm{BOR}=100 \times$ (ALS $\times$ admissions/number of beds $\times 365$ ). TI is a measure that is related to the BTO. It measures the average time that beds are unoccupied between successive patients. $\mathrm{TI}=(365 / \mathrm{BTO})-\mathrm{ALS}$.

PL technique provides a graphical method that makes use of the three indicators (BTO, BOR, and ALS) concurrently in assessing the relative performance of hospital wards. ${ }^{[6]}$ According to Figure 1, the horizontal and vertical demarcations represent the mean values of the BTO ratio and $70 \%$ BOR based on the standard of Iranian Ministry of Health. ${ }^{[8]}$

All data were analyzed by the Statistical Package for the Social Sciences software version 11.5 (SPSS Inc., Chicago, IL, USA). Continuous data are presented as mean \pm standard deviation (SD). Proportions are presented as number (\%). Comparisons between groups were analyzed using unpaired two-tailed $t$-test, Mann-Whitney test, Chi-square test, or Fisher's exact test as appropriate. Statistical significance was defined as $P<0.05$.

A total of 1719 patients were studied from March 2008 to March 2012. The mean \pm SD (median) of age and length of ICU stay were $44.28 \pm 21$ (40) and $9.16 \pm 12.9$ (4.65), respectively. More men were admitted in our medical ICU than women ( $54 \%$ vs. $46 \%$ ). The overall mortality rate was $29.1 \%$ during the period of study [Table 1]. As shown in Figure 2, the peak of admission was between $10 \mathrm{pm}$ and 4 am and the least was between 6 am to 8 am and 9 am to 11 am that could

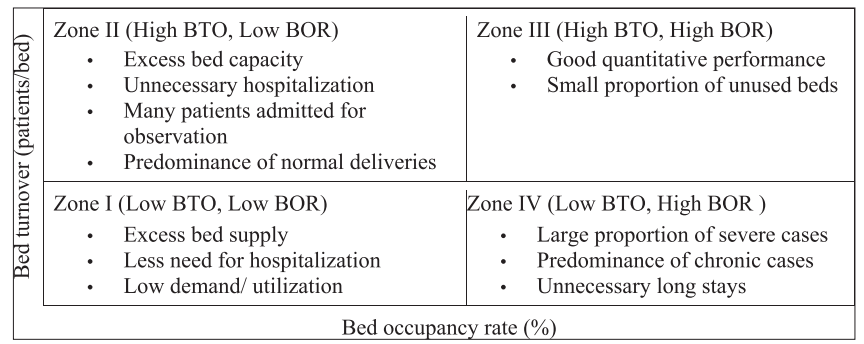

Figure 1: The Pabon Lasso's model and the interpretation of each zone (BTO; Bed turnover, BOR; Occupancy rate). be explained by the shifting time of nursing staffs. Mortality was highest between $10 \mathrm{pm}$ and $2 \mathrm{am}$. There was no significant difference in ICU mortality during different days of the week. Figure 3 shows admissions on different days of the week, with the highest admission rate on Wednesdays.

PL's graph of ICU performance showed an ascending trend from 2008 to 2010, and then it became flat to descending during 2011-2012 [Figure 4]. While during 2008-2010, the slope of the median of ICU stay increased slowly it has had sharp increment in 2012 and subsequently our medical ICU accepted fewer patients with higher occupancy rate than 2008-2011 [Table 2].

\section{Discussion}

Our study showed that nonoffice hour ICU admissions were not associated with poorer outcomes. There were no significant

\begin{tabular}{|c|c|c|c|}
\hline & Expired & Survived & $P$ \\
\hline Length of stay (days) & $9.19 \pm 13$ & $9.07 \pm 11$ & 0.870 \\
\hline Age (year) & $50.3 \pm 21$ & $41.7 \pm 20$ & $<0.001$ \\
\hline \multicolumn{4}{|l|}{ Admission } \\
\hline Holiday & 98 (28.4) & 247 (71.6) & 0.791 \\
\hline Workday & $403(29.3)$ & $971(70.7)$ & \\
\hline \multicolumn{4}{|l|}{ Sex } \\
\hline Men & $269(28.9)$ & $663(71.1)$ & 0.790 \\
\hline Women & $239(29.5)$ & $555(70.5)$ & \\
\hline \multicolumn{4}{|l|}{ Time of admission (h) } \\
\hline $7.00-13.00$ & $53(26.5)$ & $147(73.5)$ & 0.663 \\
\hline $13.00-21.00$ & $171(29.8)$ & $402(70.2)$ & \\
\hline $21.00-7.00$ & $277(29.3)$ & $669(70.7)$ & \\
\hline
\end{tabular}

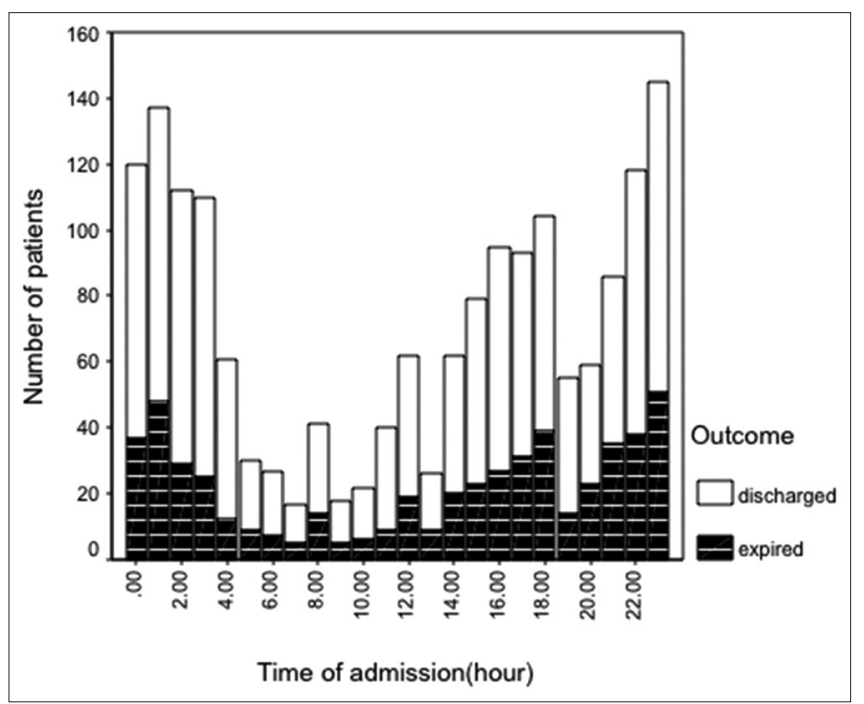

Figure 2: The frequency and distribution of admissions and mortality based on time of admission in a medical Intensive Care Unit of a teaching hospital, Shiraz, Iran, 2008-2012. 
differences in ICU mortality and length of stay between patients admitted during office hours and nonoffice hours. Moreover, statistical analyses showed that hospital mortality rates were not significantly higher on weekends, at night, or any day of the week. This implies that our ICU had a good quantitative performance $24 \mathrm{~h}$ a day, 7 days a week.

Our study showed that $20.1 \%$ of ICU admissions occurred during weekends and 55\% during nights (21:00-7:00). As a result, a remarkably high proportion $(75.1 \%)$ of patients was admitted during nonoffice hours. Other studies have reported similar findings where $65.6 \%-69 \%$ of ICU admissions occurred during weekends or at night. ${ }^{[9]}$ Appropriate management during the first few hours after admission in the ICU is crucial for the clinical outcomes of critically ill patients; it is very important to maintain a good quantitative performance during nonoffice hours.

The 2003 Society of Critical Care Medicine guidelines for adult ICUs have recommended a 24 -h in-house coverage by intensivists who are dedicated to the care of ICU patients and do not have conflicting responsibilities. ${ }^{[10]}$

Although the differences were not significant, the rate of hospital death was lower for patients admitted to the ICU

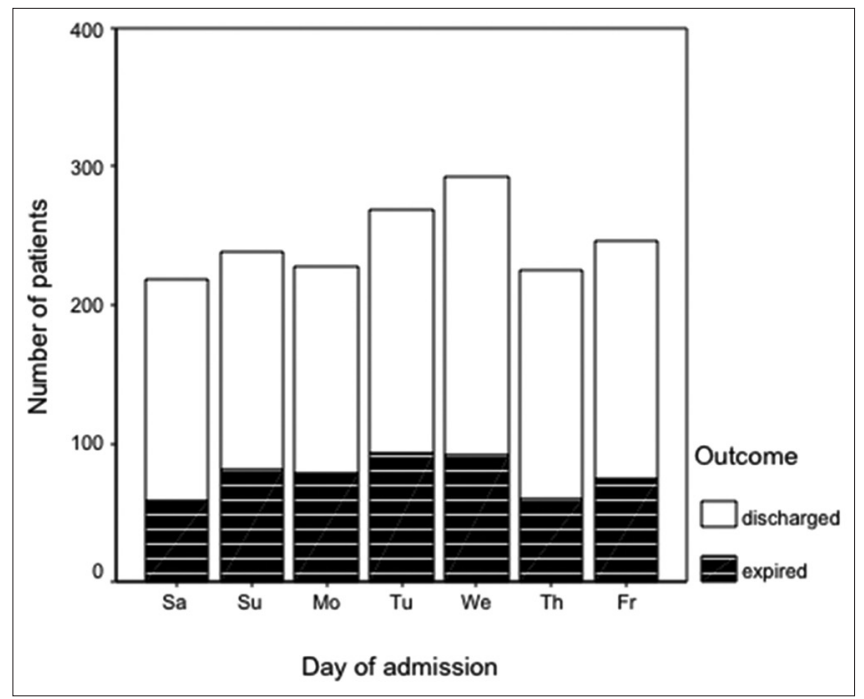

Figure 3: The frequency and distribution of admissions and mortality based on day of the week of admission to a medical ICU of a teaching hospital, Shiraz, Iran, 2008-2012. at night $(14.1 \%)$, compared to those admitted during the day $(15 \%)(P=0.417)$. Mortality was also higher during nonoffice hours during the day (13:00-21:00) compared with office hours $(7: 00-13: 00)$. On the other hand, the rate of hospital death was not higher for patients admitted to the ICU on weekends $(5.7 \%)$, compared with those admitted on weekdays $(23.4 \%)(P=0.735)$. These results could be related to the staffing pattern in our ICU where resident physicians and critical care nurses staffed the ICU in-house at constant levels every day of the week and $24 \mathrm{~h}$ a day, while dedicated intensivists led the morning rounds on every day of the week but did not stay in-house overnight. We had fewer ICU admissions (20.1\%) and no conferences or classes on weekends. With the same staffing levels, the lighter workload might have resulted in better patient care and therefore, lower hospital mortality for patients admitted on weekends.

Table 2 shows BTO, length of stay, and occupancy in our ICU throughout the 5 years of our study. According to PL's model, except for 2012, our ICU was in zone III, with a high BTO rate and a high occupancy [Figure 4]. Although the aging of population and increases in underlying comorbidities in community could prolong ICU stay, we think the main explanation for decrement in BTO in 2012 was due to changes in intensivist's team controlling ICU admissions and management. During 2008-2010, four pulmonologists were in charge for patients' admission and their management,

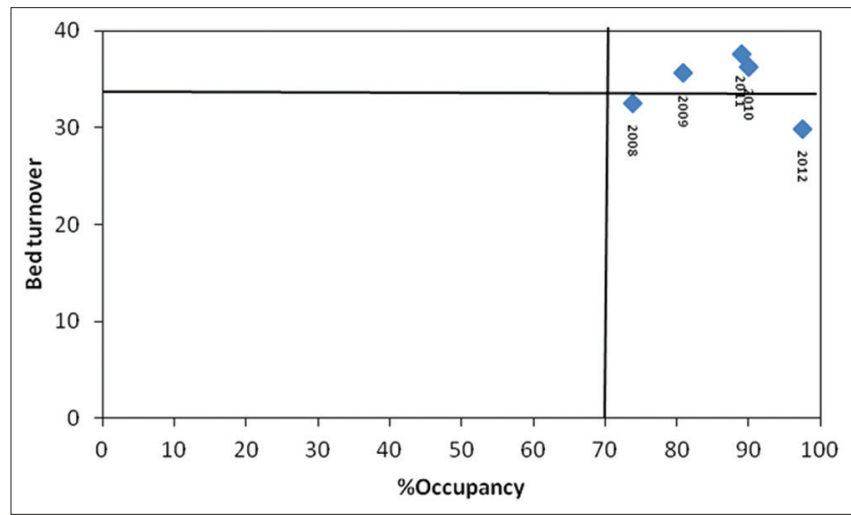

Figure 4: The status of the medical Intensive Care Unit of Shahid Faghihi Hospital, Shiraz, Iran, based on Pabon Lasso's Model 2008-2012.

Table 2: Data on the performance indicator of a medical Intensive Care Unit in a teaching hospital, Shiraz, Iran, 2008-2012

\begin{tabular}{|c|c|c|c|c|c|c|c|c|}
\hline \multirow[t]{2}{*}{ Year } & \multirow[t]{2}{*}{ Total number } & \multirow[t]{2}{*}{ Men (\%) } & \multicolumn{2}{|c|}{ Mean士SD (median) } & \multirow[t]{2}{*}{ Bed turnover } & \multirow[t]{2}{*}{ Occupancy (\%) } & \multirow{2}{*}{$\begin{array}{c}\text { Turnover } \\
\text { interval (days) }\end{array}$} & \multirow[t]{2}{*}{ Mortality (\%) } \\
\hline & & & Length of stay (days) & Age (years) & & & & \\
\hline 2008 & 331 & 54.4 & $8.58 \pm 12.6(3.84)$ & $42.47 \pm 21(38)$ & 32.50 & 73.92 & 2.93 & 23.3 \\
\hline 2009 & 357 & 54.1 & $8.36 \pm 9.6(4.60)$ & $44.31 \pm 21(40)$ & 35.60 & 80.89 & 1.96 & 25.8 \\
\hline 2010 & 362 & 56.1 & $9.01 \pm 13.5(4.86)$ & $44.65 \pm 21(42)$ & 36.30 & 90.00 & 1.01 & 36.7 \\
\hline 2011 & 377 & 51.2 & $8.55 \pm 9.9(4.72)$ & $45.52 \pm 21(42)$ & 37.60 & 89.01 & 1.07 & 31.6 \\
\hline 2012 & 292 & 55.8 & $11.76 \pm 17.9(5.47)$ & $44.22 \pm 20(38)$ & 29.90 & 97.50 & 0.31 & 27.4 \\
\hline Total & 1719 & 54.2 & $9.16 \pm 12.9(4.65)$ & $44.28 \pm 21(40)$ & 34.38 & 86.28 & 1.46 & 29.1 \\
\hline
\end{tabular}

SD: Standard deviation 
while during 2011-2012, two of them retired and two newly graduated pulmonologists were involved. Nevertheless, we had an acceptable quantitative performance in our ICU with a small proportion of unused beds.

While HIS was started in late 2007 in our hospital, the clinical information system (CIS) section has not been accepted by our physicians. Hence, data regarding the underlying comorbidities and APACHE scores were not available and we could not exactly compare the severity of diseases among our patients during the study period. Another limitation was that our study was single centered and based on a university hospital.

In conclusion, our results showed that nonoffice hour ICU admissions were not associated with poorer outcomes in a medical ICU equipped with patient management guidelines and staffed by intensivists who were on-call for $24 \mathrm{~h}$ and led the morning rounds on every day of the week, but did not stay in-house overnight. Moreover, the time of day and day of the week admissions to our ICU were not associated with significant differences in hospital mortality.

\section{Acknowledgment}

The authors thank N. Bazrafkan in the IT section of Shahid Faghihi hospital for her honest cooperation. We thank Pamela Waltl (AuthorAID in the Eastern Mediterranean) for improving the use of English in the manuscript.

\section{Financial support and sponsorship}

Nil.

\section{Conflicts of interest}

There are no conflicts of interest.

\section{REFERENCES}

1. Pronovost PJ, Angus DC, Dorman T, Robinson KA, Dremsizov TT, Young TL. Physician staffing patterns and clinical outcomes in critically ill patients: A systematic review. JAMA 2002;288:2151-62.

2. Barnett MJ, Kaboli PJ, Sirio CA, Rosenthal GE. Day of the week of intensive care admission and patient outcomes: A multisite regional evaluation. Med Care 2002;40:530-9.

3. Laupland KB, Shahpori R, Kirkpatrick AW, Stelfox HT. Hospital mortality among adults admitted to and discharged from intensive care on weekends and evenings. J Crit Care 2008;23:317-24.

4. Numa A, Williams G, Awad J, Duffy B. After-hours admissions are not associated with increased risk-adjusted mortality in pediatric intensive care. Intensive Care Med 2008;34:148-51.

5. Morales IJ, Peters SG, Afessa B. Hospital mortality rate and length of stay in patients admitted at night to the Intensive Care Unit. Crit Care Med 2003;31:858-63.

6. Pabón Lasso H. Evaluating hospital performance through simultaneous application of several indicators. Bull Pan Am Health Organ 1986;20:341-57.

7. Masoompour SM, Petramfar P, Farhadi P, Mahdaviazad H. Five-year trend analysis of capacity utilization measures in a teaching hospital 2008-2012. Shiraz E Med 2015;16:e21176.

8. Ministry of Health and Medical Education. Standard Guidelines and Criteria of Evaluation of Public Hospitals in the Country. Tehran: Ministry of Health and Medical Education Publication; 2007.

9. Romo H, Amaral AC, Vincent JL. Effect of patient sex on Intensive Care Unit survival. Arch Intern Med 2004;164:61-5.

10. Fowler RA, Sabur N, Li P, Juurlink DN, Pinto R, Hladunewich MA, et al. Sex-and age-based differences in the delivery and outcomes of critical care. CMAJ 2007;177:1513-9. 